\title{
Emerging dynamics of macrophage cellular senescence and immunosenescence in governing organismal aging and disease: Concepts and opportunities
}

\author{
Rohit Sharma*, Ph.D. \\ *Faculty of Applied Sciences \& Biotechnology, Shoolini University, Solan, 173229, India.
}

Correspondence to: Dr Rohit Sharma

Assistant Professor (INSPIRE Faculty Fellow)

Faculty of Applied Sciences \& Biotechnology,

Shoolini University, Solan, 173229, India.

rohit25sharma@gmail.com

rohit.sharma@shooliniuniversity.com

\begin{abstract}
An intricate relationship between impaired immune functions and the age-related accumulation of tissue senescent cells (SC) is rapidly emerging. The immune system is unique as it undergoes mutually inclusive and deleterious processes of immunosenescence and cellular senescence with advancing age. While factors inducing immunosenescence and cellular senescence may be shared, however, both these processes are fundamentally different which holistically influence the aging immune system. Immunosenescence is a well-characterized phenomenon, but our understanding and biological impact of cellular senescence in immune cells, especially in the innate immune cells such as macrophages, is only beginning to be understood. Tissue-resident macrophages are longlived, and while functioning in tissue-specific and niche-specific microenvironments, senescence in macrophages can be directly influenced by senescent host cells which may impact organismal aging. In addition, evidence of age-associated immunometabolic changes as drivers of altered macrophage phenotype and functions such as inflamm-aging is also emerging. The present review describes the emerging impact of cellular senescence vis-à-vis immunosenescence in aging macrophages, its biological relevance with other senescent non-immune cells, and known immunometabolic regulators. Gaps in our present knowledge, as well as strategies aimed at understanding cellular senescence and its therapeutics in the context of macrophages, have been reviewed.
\end{abstract}

Keywords: Macrophages, Senescence, Immunosenescence, Immunometabolism, Aging

Abbreviations:

SC: senescent cells

SASP: senescence-associated secretory phenotype

SA- $\beta$-gal: senescence-associated $\beta$-galactosidase

mTOR: mammalian target of rapamycin

ROS: reactive oxygen species

NAD: nicotinamide adenine dinucleotide 


\section{Introduction}

The last decade has seen rapid progress in our understanding of the molecular etiology and pathological effects of aging. There is increasing evidence that the stochastic phenomenon of aging is essentially a culmination of cellular and molecular damage over time that gradually manifests as the visible macrophenotype of aging (Bhatia-Dey et al., 2016; Chandrasekaran et al., 2017). Several different theories were formulated to explain the causes and mechanisms of aging, but gerontologists are now showing considerable interest in the cellular senescence centric interpretation of aging which is beginning to uncover an integrative and tangible understanding of the known effects of aging (Dodig et al., 2019; Borghesan et al., 2020). Aging itself is not a disease, but it rather predisposes elderly to several age-associated disorders including the characteristic hallmark diseases of $21^{\text {st }}$ century, i.e., cancer and diabetes (Huo et al., 2018; DeSantis et al., 2019). In fact, a view is now emerging that the various age-related diseases should be studied in the purview of cellular senescence, and that targeting cellular senescence itself could be a novel approach in preventing frailty and the aggravation of a number of age-associated disorders (Borghesan et al., 2020; Report, 2021). This is supported by several studies which have demonstrated that accumulation of SC shorten healthspan and lifespan (Baker et al., 2016), and delaying the development of cellular senescence (Sharma et al., 2021) or lifelong removal of SC can enhance longevity (Baker et al., 2011), improve tissue regeneration (Krishnamurthy et al., 2006) and prevent the development of chronic age-associated disorders (Palmer et al., 2019; Lewis-McDougall et al., 2019; Cai et al., 2020; Ogrodnik et al., 2021). In fact, the targeted removal of SC using senolytics is an emerging area of research, and active preclinical search, as well as clinical assessment of putative senolytics is presently underway (Xu et al., 2018; Hickson et al., 2019; Wissler Gerdes et al., 2020).

The immune system is a complex, yet coordinated and highly plastic network of cells which has been traditionally recognized to protect the integrity of the organism from invading pathogens. However, it is now understood that the pattern recognition receptors of immune cells not only recognize the pathogen-associated molecular patterns, but also respond to cell-intrinsic damageassociated molecular patterns or DAMPs suggesting their broader and more significant physiological role (Mogensen, 2009). The innate and adaptive branches of the immune system communicate either directly or through a variety of signaling molecules, while the integrated neuroendocrine-immune system governs the interactions between immune cells and other regulatory systems. As observed with different physiological responses, the immune system also undergoes characteristic functional and phenotypical deterioration with age resulting in feeble response to pathogens and vaccination, and the development of chronic age-associated inflammaging (Aw et al., 2007). These changes have been loosely described as 'immunosenescence', although whether it is the cause or a consequence of aging is still unclear. Aging in immune cells has traditionally been considered within the purview of immunosenescence; however, similar to other mammalian cells, immune cells are also liable to be affected by the different facets of cellular senescence which can have potentially deleterious impact on immune functions (Vicente et al., 2016). Although the triggers of immunosenescence and cellular senescence may overlap, but both these processes show fundamentally distinct characteristics, and yet have the potential to impair immune functions with age (Burton and Stolzing, 2018) (Table 1). However, unlike immunosenescence, our understanding of the role, and particularly the biological impact of cellular senescence in immune cells is only emerging. This is especially relevant for innate immune cells such as macrophages which form the basis of parenchymal homeostasis, integrity, and perform unique tissue-specific functions as resident immune cells. This review describes our present 
understanding and relevance of cellular senescence in immune cells per se, with an emphasis on macrophages. The lacunae in our present knowledge, as well as the potential for further research is discussed to understand how senescent macrophages contribute to the aging process.

Table 1: Selected markers of immunosenescence and cellular senescence in macrophages

\begin{tabular}{ll}
\hline Immunosenescence & Cellular senescence \\
\hline Decreased activation response to LPS/IFN- $\gamma$ & $\begin{array}{l}\text { Cell cycle arrest; Persistent p53/p21 and/or p16 } \\
\text { expression } \\
\text { DNA damage }\end{array}$ \\
Impaired chemotaxis & Telomere attrition \\
Decreased cytokine response & Presence of SASP \\
Impaired wound healing & Hypertrophy \\
M1/M2 polarization & Reduced cell proliferation \\
Impaired antigen presentation & Increased SA- $\beta$-gal activity \\
ROS burden* & ROS burden* \\
Metabolic alterations* & Metabolic alterations* \\
\hline
\end{tabular}

*Overlapping parameters

\section{Cellular senescence, immunity and aging: emerging concepts}

The fact that mitotic mammalian cells fail to divide after a certain finite number of cell divisions in vitro was a seminal discovery in geroscience (Hayflick and Moorhead, 1961). The socalled 'Hayflick limit' seemingly provided a link between cell division and organismal aging, but this phenomenon remained controversial and was initially dismissed as a mere in vitro artifact (Hayflick, 1998; de Magalhães, 2004). However, multiple studies have now confirmed that SC play a causal role in governing organismal aging and age-dependent disorders (Campisi and Robert, 2014; Childs et al., 2015; Katzir et al., 2021), and in fact, cellular senescence is established as one of the well-studied hallmarks of aging (López-Otín et al., 2013). Cellular senescence is characterized by cellular hypertrophy, permanent cell cycle arrest by the activation of cell cycle inhibitory pathways such as $\mathrm{p} 53 / \mathrm{p} 21^{\mathrm{WAF} 1}$ and/or $\mathrm{p} 16^{\mathrm{Ink} 4 \mathrm{a}} / \mathrm{pRb}$, resistance to apoptosis by deregulation of Bax/Bcl-2 signaling, telomere attrition, development of a senescence associated secretory phenotype or SASP, and altered metabolic longevity pathways such as SIRT and mTOR (Carroll and Korolchuk, 2018; Hernandez-Segura et al., 2018; Maduro et al., 2021; Kumari and Jat, 2021). The induction of cellular senescence is intimately linked to external and intrinsic stressors. Age-dependent decline in cell stress response capacity results in impaired redox balance, augmentation of genotoxic stress and DNA damage that ultimately aids in cell cycle arrest and the development of cellular senescence (Robinson et al., 2018; Chen et al., 2020). Moreover, there is evidence that the characteristic telomere attrition, as observed in $\mathrm{SC}$, could also be accelerated by 
oxidative damage (von Zglinicki, 2002; Ludlow et al., 2014). However, it is important to consider that SC are naturally occurring cells and their presence per se does not reflect on the pathological state of the organism, since SC are essential for processes such as wound healing and repair (Demaria et al., 2014). In younger organisms, SC are constantly replaced, but as we age, SC turnover decreases and tissue SC burden rapidly increases which contributes to age-related pathologies (Karin et al., 2019). More significantly, accumulation of SC result in the chronic presence of SASP which has pro-tumorigenic and pro-inflammatory paracrine effects on nearby healthier cells (Coppé et al., 2010). Thus, cellular senescence appears to be an antagonistic hallmark of aging and a candidate example of antagonistic pleiotropy theory of aging.

The exact causes of SC accumulation with age are yet to be completely understood. In this regard, the role of immune cells in maintaining SC cell homeostasis in tissues is being increasingly recognized (Prata et al., 2018). The SASP components of SC serve to chemotactically attract various immune cells such as macrophages, NK cells and cytotoxic T cells which subsequently recognize the upregulated immunogenic ligands on SC (such as MICA and ULBP2) resulting in their efficient removal (Coppé et al., 2010; Sagiv et al., 2016; Hall et al., 2016). That SC accumulate in aging organisms, could at least in part be ascribed to deficient immune functions/immunosurveillance associated with immunosenescence and/or cellular senescence (Figure 1). Indeed, a recent work showed that mice deficient in the cytotoxic potential of immune cells exhibited both higher SC tissue burden and chronic inflammation suggesting that weakened immunosurveillance could be a critical aspect regulating SC accumulation in vivo (Ovadya et al., 2018). Another study showed that impaired immunosurveillance in CD4+T cells results in the development of murine hepatocellular carcinomas, thus suggesting that senescence surveillance is important for tumor suppression in vivo (Kang et al., 2011). Similarly, it was reported that NK cells play a critical role in eliminating senescence activated stellate hepatic cells both in vitro and in vivo (Krizhanovsky et al., 2008). The role of macrophages in the removal of SC has long been recognized (Kay, 1975; Mevorach et al., 2010). Using a co-culture system, a recent study showed that macrophages can induce TNF- $\alpha$ mediated apoptosis in senescent fibroblasts followed by their phagocytic clearance (Ogata et al., 2021). Macrophages are also involved in the elimination of SC in the uterine stroma surrounding the embryo implantation site following parturition in mice (Egashira et al., 2017). In addition, the SASP of senescent hepatic cells not only attracts macrophages (Irvine et al., 2014), but also influences the M1/M2 polarization state of macrophages (Lujambio et al., 2013). It is also interesting to note that SC themselves can also develop features of immune evasion. It was demonstrated that $\mathrm{SC}$ can escape the immune system by upregulating the expression of non-classical MHC molecule HLA-E, which inhibits NK cells and CD8+ T cells mediated immune responses against SC, thereby aiding in inefficient removal and accumulation 
of SC (Pereira et al., 2019). This potentially suggests autonomous functioning of SC, much in line with tumorous cells and requires further exploration.

Figure 1: Immune cells mediated removal of SC is affected by cellular senescence and immunosenescence. In young organisms, SC secrete SASP factors which attract innate and adaptive immune cells resulting in the removal of SC. During aging, immunosenescence and cellular senescence collectively impair several immune functions which may together contribute to the inefficient clearance of SC and their gradual accumulation.

\section{Macrophage senescence: culmination of immunosenescence and cell senescence?}

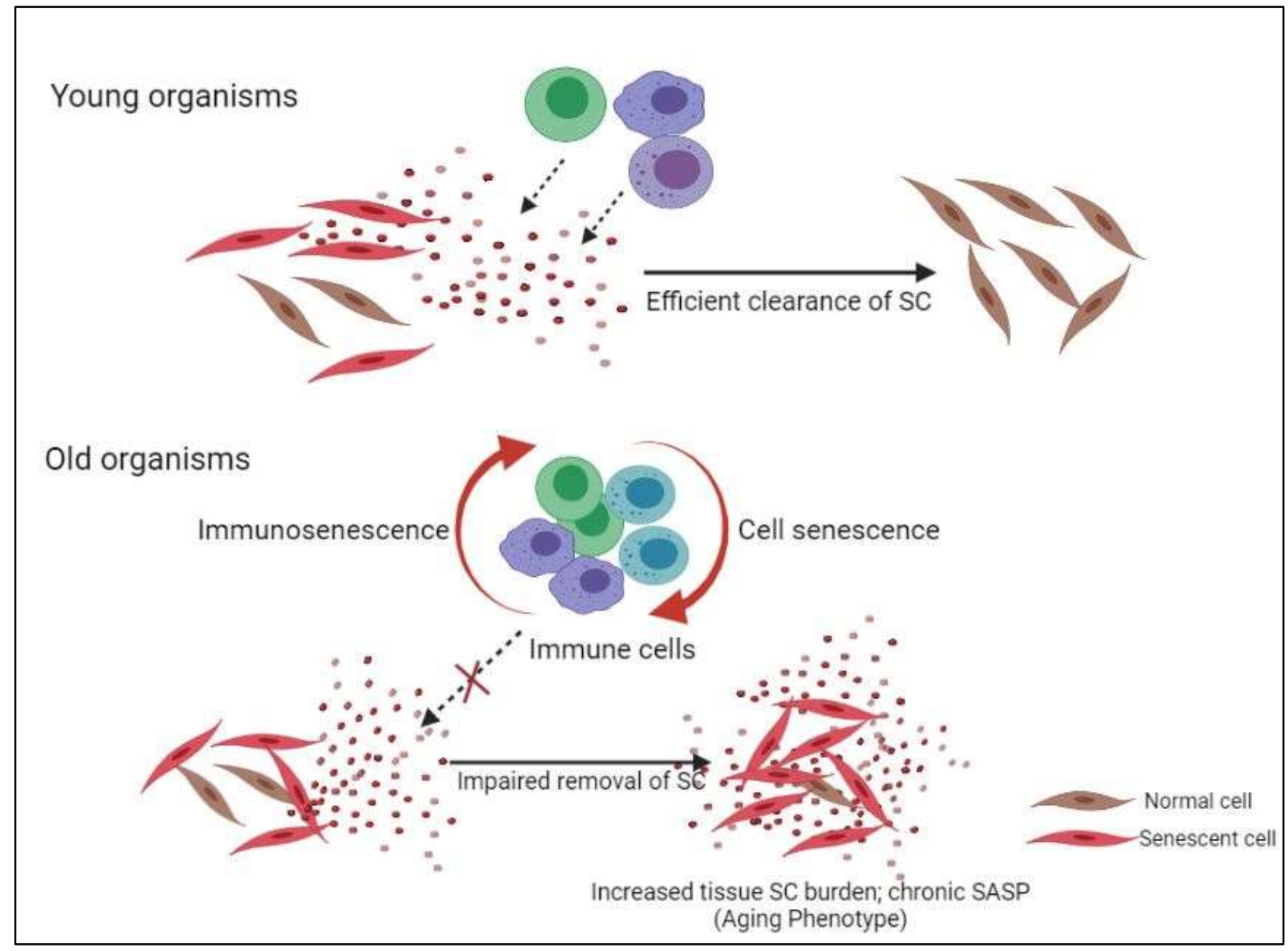

The immune system reacts to exogenous and endogenous threats by initiating an inflammatory response. Macrophages are involved both during the onset of inflammation as well as its successful resolution. The recognition of a foreign particle prompts proinflammatory behavior in macrophages (also called classical activation or M1) characterized by increased production of inflammatory cytokines, respiratory burst and phagocytosis (Orecchioni et al., 2019). To resolve the latent inflammation and debris, macrophages switch to an anti-inflammatory M2 phenotype by initiating reconstruction of damaged tissue and by prompting synthesis of extracellular matrix and cell growth (Rőszer, 2015). As antigen presenting cells, macrophages are involved in the regulation and differentiation of adaptive immune responses, while scavenger receptors on macrophages recognize and remove oxidized proteins/lipoproteins expressing damaged or apoptotic cells (Krieger and Herz, 1994; Schliehe et al., 2011). These diverse functions of 
macrophages are severely hampered with age and are often attributed to characteristic immunosenescence. For instance, there are reports suggesting that M1/M2 polarization of macrophages is affected during aging, although the nature of this skewness is debatable. Agedependent M1 to M2 polarization of macrophages has been observed in aging skeletal muscles (Cui et al., 2019) and during macular degeneration (Zandi et al., 2015), while we previously observed an anti-inflammatory phenotype in aged animals (Sharma et al., 2014). On the other hand, an age-dependent shift towards M1 pro-inflammatory state was also observed in aged mice which was ascribed to the degeneration of enteric nervous system (Becker et al., 2018). These observed differences could be attributed to the heterogeneity and plasticity of macrophages, but regardless, any perturbations in the normal M1/M2 balance in macrophages may dysregulate the development of the host response, making elderly more susceptible to inflammatory and infectious diseases (Mahbub et al., 2012; Wynn et al., 2013). Studies have also shown that macrophage phagocytic ability (Wong et al., 2017), TLR expression (Renshaw et al., 2002) and LPS-induced cytokine production declines with age (Chelvarajan et al., 2006). Importantly, it was also identified that age-related decrease in phagocytic ability was not observed in bone marrow derived macrophages and monocytes suggesting that age-related changes in macrophage functions might not be intrinsic, but could be related to external environmental factors (Linehan et al., 2014). Aging also results in decreased antigen presenting capacity of macrophages which may compromise the swiftness and the potency of adaptive immune response. Studies have shown that old mice showed impaired stimulation as well as expression of MHC class II molecules compared to macrophages from young mice after stimulation with IFN- $\gamma$ (Davila et al., 1990; Herrero et al., 2001). Aging has also been shown to reduce macrophage mediated tissue repair and regeneration both in mice and humans. It was observed that local injection of peritoneal macrophages isolated from young mice could accelerate would healing in older animals (Danon et al., 1989). Age-related shifts in macrophage infiltration into wounds, alterations in chemokine content, and a concurrent decline in wound macrophage phagocytic function was also documented which may contribute to the delayed repair response during aging (Swift et al., 2001). A recent study showed that injured arteries in aging rats develop thicker neointimas, which were significantly correlated with a higher number of tissue macrophages and increased vascular IL-18 (Rodriguez-Menocal et al., 2014). Further, in vivo depletion of macrophages by clodronate liposomes ameliorated the vascular accumulation of IL-18 and development of thicker neointimas (Rodriguez-Menocal et al., 2014).

Unlike immunosenescence, conclusive evidence of cellular senescence in macrophages is only beginning to be elucidated. Prior studies in T cells have shown that immune cells $d o$ exhibit the features of cellular senescence both in vitro and in vivo, while such work on macrophages remained scanty (Vallejo et al., 1999; Effros, 2004; Chou and Effros, 2013). Nonetheless, in vitro studies have shown that on exposure to external stressors, macrophages can develop senescence like features including p21/p53 mediated cell cycle arrest (Nyunoya et al., 2003; Fong et al., 2007), altered morphology and the development of SA- $\beta$-gal activity (Kim et al., 2015). In a recent study, we observed that peritoneal macrophages isolated from old mice exhibit heterogenous and elongated morphology, enhanced expression of cell cycle inhibitors $\mathrm{p} 53 / \mathrm{p} 21^{\mathrm{WAF} 1} / \mathrm{p} 16^{\text {Ink4a }}$, and SA$\beta$-gal activity as compared to macrophages isolated from younger animals (Kumar et al., 2020). In another study, age-related increase in the characteristic marker of senescence $\mathrm{p} 16^{\text {Ink4a }}$ inhibited the normal phenotype of adipose tissue macrophages and contributed to type II diabetes risk (Fuentes et al., 2011). A negative correlation between Ki67 proliferation marker and cellular senescence marker SA- $\beta$-gal expression was observed in cultured macrophages suggesting that SA- $\beta$-gal activity labels macrophages with decreasing proliferation tendency (Holt and Grainger, 2012). A 
recent study observed that the majority of $\mathrm{p} 16^{\mathrm{Ink} 4 \mathrm{a}}$ and SA- $\beta$-gal positive cells in the tissues of aging mice were, in fact macrophages, indicating a significant role of senescent macrophages which previously had been attributed to non-immune SC only (Hall et al., 2016). However, in their follow up work, the same authors argued that $\mathrm{p} 16^{\text {Ink4a }}$ and SA- $\beta$-gal expression may be unrelated to senescence, and may only be a part of a reversible response of macrophages to their physiological stimuli (Hall et al., 2017). Altogether, these observations suggest an unexpected role of $\mathrm{p} 16^{\text {Ink4a }}$ expression in macrophages which warrants deeper characterization vis-à-vis other markers of cellular senescence. However, another hallmark indicator of cellular senescence, i.e., telomere attrition, has also been observed in macrophages both during aging and disease. Analyses of bone marrow-derived macrophages showed strong age-dependent shortening of telomere length in aged (19-24-mo-old) mice as compared to young (6-wk-old) mice (Sebastián et al., 2009). Murine peritoneal macrophages isolated from first-generation telomerase reverse transcriptasedeficient mice significantly upregulated SA- $\beta$-gal activity and the expression of genes regulating cell cycle arrest such as p16, p21, and the retinoblastoma protein (Gizard et al., 2011). Mean telomere length in peripheral blood leukocytes was significantly shorter in sickle cell disease patients which directly correlated with the disease genotype and inflammation markers (Colella et al., 2017). Some studies have suggested that a characteristic SASP is also prevalent in macrophages. A recent report has shown that LPS treated THP-1 macrophages develop features of cellular senescence and SASP as evident by expression of p53/p21/p16 cell cycle inhibitors and various SASP components (Wang et al., 2020). Using a diabetic mice model, it was observed that macrophages in diabetic kidneys showed archetypal features of cellular senescence as evident from increased mRNA expression pertaining to cell cycle arrest (p16/p21) and SASP (IL-6, TGF- $\beta$, PAI-1, MCP-1) suggesting that senescent macrophages are prevalent in vivo and could be a potential source of SASP in disease condition (Prattichizzo et al., 2018).

It is thus reasonable to envisage that both cellular senescence and immunosenescence can impact macrophage functions. However, either a conclusive correlation or a causative linkage between cellular senescence and immunosenescence in macrophages or other immune cells has not been established yet. Nonetheless, studies on telomere shortening have highlighted multiple negative effects on immune functions and disease which could be related to some aspects of immunosenescence. For example, telomerase knockout mice developed exaggerated lung inflammation and increased mortality upon respiratory staphylococcal infection (Kang et al., 2018). Authors observed that telomere dysfunction caused macrophage mitochondrial abnormality, oxidative stress, and hyperactivation of the NLRP3 inflammasome, suggesting that loss of telomeres is sufficient to drive mitochondria mediated redox imbalance, and thus contribute to macrophage functional incapacity which could be directly related to immunosenescence (Kang et al., 2018). Similarly, using telomerase knockout mice, it was observed that telomere loss is the cause for the enhanced oxidative stress, reduced Stat5a oxidation and phosphorylation and, ultimately, for the impaired GM-CSF-dependent macrophage proliferation (Sebastián et al., 2009). It was also demonstrated that monocytes (but not lymphocytes) of aged type II diabetic patients had significantly lower mean telomere length and a significant inverse relationship between oxidative DNA damage and telomere length was observed in diabetic group (Sampson et al., 2006). Another study showed a causative link between accumulating ROS and the development of senescent phenotype in macrophages (Singh et al., 2019). Together, it appears that some degree of causation between cellular senescence and immunosenescence does exist, but further deeper studies are required to completely understand this dichotomy. 


\section{Interactions between macrophages and senescent cells}

Macrophages are present throughout the body, wherein they reside as specialized tissue resident cells and perform tissue-specific and niche-specific functions. As a result, different tissue resident macrophages may be functionally distinct, and respond differentially to effector functions (Minutti et al., 2017; Lacerda Mariano et al., 2020). Moreover, in terms of cellular senescence, recent studies have shown that different aging tissues experience varying degree of SC burden as the rate of cellular senescence is dependent on both the age of tissue, as well as the type of tissue (Karin et al., 2019; Tuttle et al., 2020; Sharma et al., 2021). Thus, it is conceivable that dynamic interactions between specialized long-lived macrophages and host tissues with varying degree of cellular senescence, may differentially influence their functional capacity and/or the rate of cellular senescence (Figure 2). Although these aspects are less explored, our previous ex vivo study showed that the secretory phenotype of senescent preadipocytes, but not proliferating preadipocytes, could induce a senescence-like phenotype in peritoneal macrophages isolated from young animals (but not from old animals) characterized by a strong SA- $\beta$-gal activity, cellular hypertrophy, increase in $\mathrm{p} 16^{\text {Ink4a }}$ expression and intracellular ROS production (Kumar et al., 2020). This implies that in a heterogenous and dynamic in vivo system, the highly senescence-prone adipose tissue (Minamino et al., 2009; Sharma et al., 2021) could directly influence cellular senescence in adipose tissue macrophages. A recent study showed that macrophages induced apoptosis in senescent fibroblasts and prevented their accumulation, however, chronic presence of fibroblast SASP factors downregulated macrophage mediated apoptosis and phagocytosis thereby potentially suggesting that senescent tissues can affect macrophage functions which can contribute to increased tissue SC burden (Ogata et al., 2021). Similarly, another report identified that lysophosphatidylcholines expression is elevated in the phospholipids of senescent dermal fibroblasts which interfere with the toll-like receptor 2 and 6/CD36 signaling and subsequent phagocytic capacity in macrophages (Narzt et al., 2021). Exposure of macrophages to the secretome of senescent hepatocytes induced an M2 phenotype, while an M1 phenotype was observed in macrophages exposed to secretory factors of non-senescent hepatocytes (Sen et al., 2021). Similarly, exposure of macrophages to the conditioned media of senescent thyrocytes induced an anti-inflammatory M2 phenotype in macrophages showing high CD206 and low MHC II markers, and upregulated CCL17 secretion which ultimately promoted tumor progression (Mazzoni et al., 2019). It is thus evident that senescent cells can directly influence the functional and phenotypical aspects of aging in macrophages which can accelerate SC burden in tissues. 


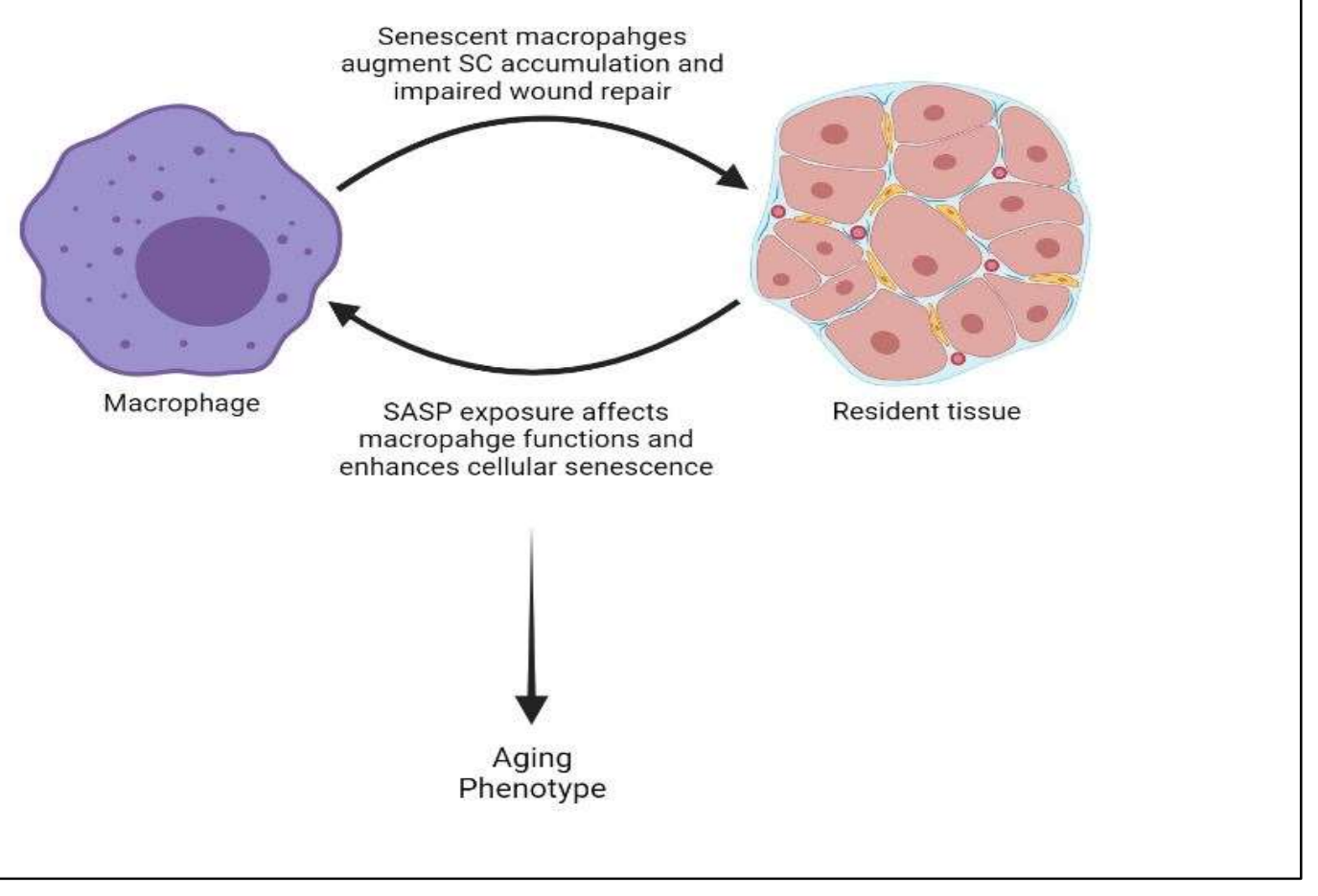

Figure 2: Bidirectional relationship between resident macrophages and tissues. Dynamic interactions between senescent host tissues and long-lived tissue resident macrophages may impact senescence and aging. SASP of host tissues can modulate macrophage functions and decrease its potency to remove damaged cells. Senescent macrophages can directly augment SC accumulation owing to impaired immunosurveillance.

\section{Immunometabolism and macrophage senescence}

Understanding the metabolic regulation of immune functions, especially during aging, is an emerging area of research (Lee et al., 2021). To precisely perform their diverse functions, immune cells like macrophages need to quickly shift between various activation states which are subject to tight regulation by metabolic pathways and nutrient availability. For instance, upon activation, M1 like macrophages rapidly convert arginine to nitric oxide through inducible NO synthase activity, while M2 macrophages convert arginine to ornithine for effector functions (Galván-Peña and O'Neill, 2014). Several studies suggest that nutrient signaling longevity pathways, such as mTOR, SIRT and Insulin/IGF-1 are the key modulators and therapeutic targets of health and lifespan (Mazucanti et al., 2015). Aged macrophages generally show heightened inflammatory metabolic pathways, despite reduced phagocytic ability, antigen presentation and mitochondrial dysfunction (van Beek et al., 2019; Lee et al., 2021). Using mass spectrometrybased proteomic approach, age-dependent alterations in inflammatory signaling, mitochondrial function, and cellular metabolic pathways were observed in aged microglia cells, while a component of the mTORC2 complex was identified as a novel upstream regulator of several biological functions (Flowers et al., 2017). Rapamycin-induced inhibition of mTOR signaling accelerated diabetic encephalopathy in rats through the augmentation of macrophage autophagy (Wang et al., 2018). Knockdown of SIRT1 in the mouse macrophage RAW264.7 cell line and in intraperitoneal macrophages broadly activated the JNK and IKK inflammatory pathways, and 
increased LPS-stimulated TNF $\alpha$ secretion (Yoshizaki et al., 2010). On the other hand, overexpression of SIRT1 during bone marrow-derived macrophage differentiation increased their proliferative capacity and self-renewal (Imperatore et al., 2017). Sirtuin family of enzymes depend on the coenzyme NAD for their activation and function. NAD levels decline with age in different tissue types, including macrophages, which have been shown to increase inflammation and impair oxidative metabolism (Minhas et al., 2019). It is plausible that declining NAD levels in aged macrophages could also compromise SIRT activity that may negatively impact inflammatory homeostasis during aging (He et al., 2020). Interestingly, senescent macrophages have recently been implicated in the immunometabolism of NAD (Covarrubias et al., 2020). It was observed that NAD consuming CD38+ M1 macrophages with upregulated markers of senescence (p16/p21) and inflammation accumulated in adipose tissue which were primarily responsible for decreased NAD availability in tissues during aging, suggesting a causative relationship between CD38+ macrophages, NAD consumption, Sirtuins and aging (Covarrubias et al., 2020). In addition, M2 phenotype macrophages were identified as novel sources of IGF1, and ablation of IGF1 receptors from myeloid cells reduced phagocytosis, increased macrophages in adipose tissue, elevated adiposity, lowered energy expenditure, and led to insulin resistance in mice fed a high-fat diet indicating that IGF1 signaling shapes the macrophage-activation phenotype (Spadaro et al., 2017). A recent study showed the regulatory role of IGF1 signaling in cellular senescence and macrophage polarization wherein mice deficient for IGF1 receptors showed attenuated premature senescence in pneumocytes, reduced M2 macrophage polarization and lung fibrosis (Chung et al., 2020). Together, it is evident that understanding the metabolic control of cellular senescence mediated by nutrient sensing pathways is essential for completely comprehending the etiology and effects of macrophage senescence.

\section{Unanswered questions and niche research areas}

There are several lacunae in our present understanding of the causes and biological effects of cellular senescence in macrophages. This is of particular importance since tissue resident macrophages, similar to memory T cells, are considered to be long lived (Bain et al., 2016), and are thus liable to accumulate cellular and biochemical insults over time leading to genotoxic stress and the development of cellular senescence (Sedelnikova et al., 2004). Some of the key aspects to be investigated are:

- The extent and depth of in vivo cellular senescence in macrophages is unclear. There is a need to characterize macrophages for age-dependent as well as tissue-dependent changes pertaining to the development of cellular senescence using the established markers of immune cell senescence (Zhou et al., 2021). Given the high heterogeneity amongst macrophages, using multi-omics approach at single cell resolution would be a prudent approach.

- Macrophage secretory phenotype changes with age, but whether it is related to the SASP of non-immune cells, or if senescent macrophage secretome contributes to inflamm-aging remains to be fully understood. In this context, whether senescent macrophages play any direct role in driving age-related inflammatory disorders such as type II diabetes or osteoarthritis is also unknown.

- More information is required to understand the interrelationship between tissue resident macrophages and SC. How SC or their secretome influence immunosenescence and/or 
cellular senescence in macrophages in vivo, and whether senescent macrophages can also impact the development of senescence in non-immune cells remains to be explored.

- Immunometabolism of senescent macrophages is also poorly studied. Whether the longevity pathways play a similar role in macrophage senescence, and if these pathways could be therapeutic targets regulating macrophage functions during aging and disease remains to be elucidated.

- Much information about the mechanisms and ligands of macrophage mediated immunosurveillance is not known. It needs to be determined whether senescent macrophages preserve their capacity of immunosurveillance which can directly impact tissue SC accumulation.

- Finally, nutraceuticals such as probiotics and polyphenols, as well as micronutrients are known to affect immunosenescence (Sharma et al., 2013; Wu et al., 2019), but whether the underlying mechanisms are relatable to cellular senescence is little understood (Sharma and Padwad, 2020) (Figure 3). In this regard, our previous work showed that green tea catechin EGCG can strongly attenuate the development of senescence-like features such as expression of $\mathrm{p} 53 / \mathrm{p} 21^{\mathrm{WAF} 1} / \mathrm{p} 16^{\text {Ink4a }}$, and SA- $\beta$-gal activity in macrophages exposed to conditioned media of senescent preadipocytes (Kumar et al., 2020). This suggests that nutraceuticals can impact senescence induction in macrophages, although further elaborative studies are warranted.

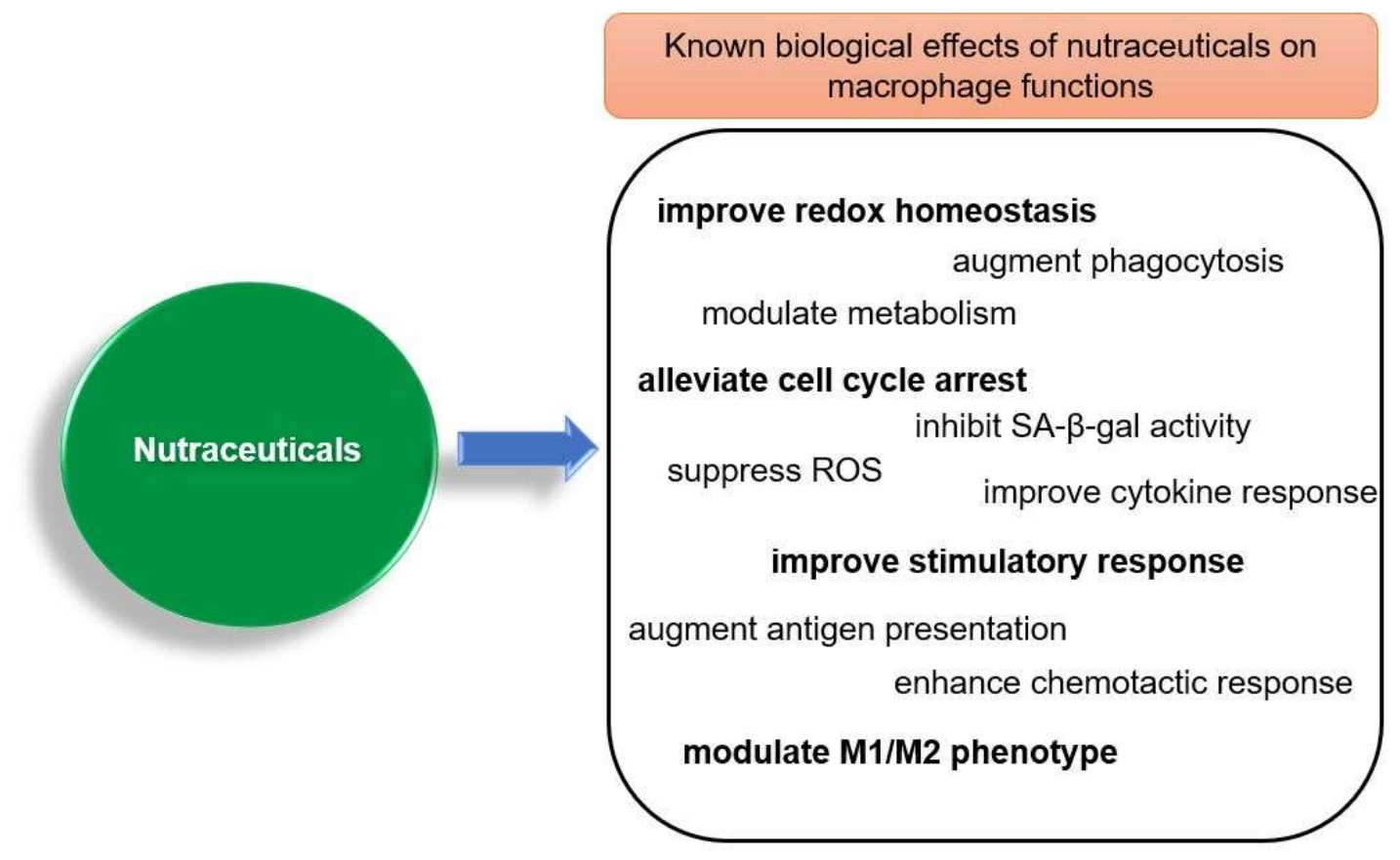

Figure 3: Multifaceted effects of nutraceuticals in modulating different aspects of cellular senescence and immunosenescence. 


\section{Concluding remarks}

Aging, cellular senescence and immunosenescence appear to be intimately linked (Sharma and Padwad, 2020). As most diverse and plastic immune cells, delineating the dichotomy of cellular senescence and immunosenescence in macrophages is necessary for comprehending the relationship between aging and immune functions. Evidence that cellular senescence is an active force driving the aging of macrophage and which may contribute to age-related pathologies is already emerging. In addition, senescent non-immune cells can also impair macrophage immune functions through paracrine effects and augment SC burden suggesting an intricate relationship between host tissues and macrophages senescence which needs further characterization. In short, it is imperative that future studies consider macrophages in the purview of cellular senescence which may ultimately provide an integrative and deeper understanding of organismal aging and the pathology of age-dependent diseases.

\section{Funding sources}

This work was supported by grant from the Department of Science \& Technology, Government of India under the INSPIRE Faculty scheme (grant no. IFA17-LSPA79).

Declarations of interest: none

\section{References}

Aw, D., Silva, A.B., Palmer, D.B., 2007. Immunosenescence: emerging challenges for an ageing population. Immunology 120, 435-446.

Bain, C.C., Hawley, C.A., Garner, H., Scott, C.L., Schridde, A., Steers, N.J., Mack, M., Joshi, A., Guilliams, M., Mowat, A.M., Geissmann, F., Jenkins, S.J., 2016. Long-lived selfrenewing bone marrow-derived macrophages displace embryo-derived cells to inhabit adult serous cavities. Nat Commun 7, ncomms 11852.

Baker, D.J., Childs, B.G., Durik, M., Wijers, M.E., Sieben, C.J., Zhong, J., Saltness, R.A., Jeganathan, K.B., Verzosa, G.C., Pezeshki, A., Khazaie, K., Miller, J.D., van Deursen, J.M., 2016. Naturally occurring p16(Ink4a)-positive cells shorten healthy lifespan. Nature 530, 184-189.

Baker, D.J., Wijshake, T., Tchkonia, T., LeBrasseur, N.K., Childs, B.G., van de Sluis, B., Kirkland, J.L., van Deursen, J.M., 2011. Clearance of p16Ink4a-positive senescent cells delays ageing-associated disorders. Nature 479, 232-236.

Becker, L., Nguyen, L., Gill, J., Kulkarni, S., Pasricha, P.J., Habtezion, A., 2018. Age-dependent shift in macrophage polarisation causes inflammation-mediated degeneration of enteric nervous system. Gut 67, 827-836.

Bhatia-Dey, N., Kanherkar, R.R., Stair, S.E., Makarev, E.O., Csoka, A.B., 2016. Cellular Senescence as the Causal Nexus of Aging. Frontiers in genetics 7, 13-13.

Borghesan, M., Hoogaars, W.M.H., Varela-Eirin, M., Talma, N., Demaria, M., 2020. A Senescence-Centric View of Aging: Implications for Longevity and Disease. Trends Cell Biol. 30, 777-791.

Burton, D.G.A., Stolzing, A., 2018. Cellular senescence: Immunosurveillance and future immunotherapy. Ageing Res Rev 43, 17-25.

Cai, Y., Zhou, H., Zhu, Y., Sun, Q., Ji, Y., Xue, A., Wang, Y., Chen, W., Yu, X., Wang, L., Chen, H., Li, C., Luo, T., Deng, H., 2020. Elimination of senescent cells by $\beta$ galactosidase-targeted prodrug attenuates inflammation and restores physical function in aged mice. Cell Res. 30, 574-589. 
Campisi, J., Robert, L., 2014. Cell senescence: role in aging and age-related diseases. Interdiscip Top Gerontol 39, 45-61.

Carroll, B., Korolchuk, V.I., 2018. Nutrient sensing, growth and senescence. The FEBS journal 285, 1948-1958.

Chandrasekaran, A., Idelchik, M.d.P.S., Melendez, J.A., 2017. Redox control of senescence and age-related disease. Redox Biology 11, 91-102.

Chelvarajan, R.L., Liu, Y., Popa, D., Getchell, M.L., Getchell, T.V., Stromberg, A.J., Bondada, S., 2006. Molecular basis of age-associated cytokine dysregulation in LPS-stimulated macrophages. J. Leukoc. Biol. 79, 1314-1327.

Chen, K., Shen, W., Zhang, Z., Xiong, F., Ouyang, Q., Luo, C., 2020. Age-dependent decline in stress response capacity revealed by proteins dynamics analysis. Sci. Rep. 10, 15211.

Childs, B.G., Durik, M., Baker, D.J., van Deursen, J.M., 2015. Cellular senescence in aging and age-related disease: from mechanisms to therapy. Nat. Med. 21, 1424-1435.

Chou, J.P., Effros, R.B., 2013. T cell replicative senescence in human aging. Curr. Pharm. Des. 19, 1680-1698.

Chung, E.J., Kwon, S., Reedy, J.L., White, A.O., Song, J.S., Hwang, I., Chung, J.Y., Ylaya, K., Hewitt, S.M., Citrin, D.E., 2020. IGF-1 Receptor Signaling Regulates Type II Pneumocyte Senescence and Resulting Macrophage Polarization in Lung Fibrosis. International Journal of Radiation Oncology*Biology*Physics.

Colella, M.P., Santana, B.A., Conran, N., Tomazini, V., Costa, F.F., Calado, R.T., Saad, S.T.O., 2017. Telomere length correlates with disease severity and inflammation in sickle cell disease. Rev Bras Hematol Hemoter 39, 140-145.

Coppé, J.P., Desprez, P.Y., Krtolica, A., Campisi, J., 2010. The senescence-associated secretory phenotype: the dark side of tumor suppression. Annu. Rev. Pathol. 5, 99-118.

Covarrubias, A.J., Kale, A., Perrone, R., Lopez-Dominguez, J.A., Pisco, A.O., Kasler, H.G., Schmidt, M.S., Heckenbach, I., Kwok, R., Wiley, C.D., Wong, H.S., Gibbs, E., Iyer, S.S., Basisty, N., Wu, Q., Kim, I.J., Silva, E., Vitangcol, K., Shin, K.O., Lee, Y.M., Riley, R., Ben-Sahra, I., Ott, M., Schilling, B., Scheibye-Knudsen, M., Ishihara, K., Quake, S.R., Newman, J., Brenner, C., Campisi, J., Verdin, E., 2020. Senescent cells promote tissue $\mathrm{NAD}(+)$ decline during ageing via the activation of CD38(+) macrophages. Nat Metab 2, 1265-1283.

Cui, C.-Y., Driscoll, R.K., Piao, Y., Chia, C.W., Gorospe, M., Ferrucci, L., 2019. Skewed macrophage polarization in aging skeletal muscle. Aging Cell 18, e13032.

Danon, D., Kowatch, M.A., Roth, G.S., 1989. Promotion of wound repair in old mice by local injection of macrophages. Proc. Natl. Acad. Sci. U. S. A. 86, 2018-2020.

Davila, D.R., Edwards, C.K., 3rd, Arkins, S., Simon, J., Kelley, K.W., 1990. Interferon-gammainduced priming for secretion of superoxide anion and tumor necrosis factor-alpha declines in macrophages from aged rats. FASEB J. 4, 2906-2911.

de Magalhães, J.P., 2004. From cells to ageing: a review of models and mechanisms of cellular senescence and their impact on human ageing. Exp. Cell Res. 300, 1-10.

Demaria, M., Ohtani, N., Youssef, S.A., Rodier, F., Toussaint, W., Mitchell, J.R., Laberge, R.M., Vijg, J., Van Steeg, H., Dollé, M.E.T., Hoeijmakers, J.H.J., de Bruin, A., Hara, E., Campisi, J., 2014. An essential role for senescent cells in optimal wound healing through secretion of PDGF-AA. Dev. Cell 31, 722-733. 
DeSantis, C.E., Miller, K.D., Dale, W., Mohile, S.G., Cohen, H.J., Leach, C.R., Goding Sauer, A., Jemal, A., Siegel, R.L., 2019. Cancer statistics for adults aged 85 years and older, 2019. CA Cancer J. Clin. 69, 452-467.

Dodig, S., Čepelak, I., Pavić, I., 2019. Hallmarks of senescence and aging. Biochemia medica 29, 030501-030501.

Effros, R.B., 2004. From Hayflick to Walford: the role of T cell replicative senescence in human aging. Exp. Gerontol. 39, 885-890.

Egashira, M., Hirota, Y., Shimizu-Hirota, R., Saito-Fujita, T., Haraguchi, H., Matsumoto, L., Matsuo, M., Hiraoka, T., Tanaka, T., Akaeda, S., Takehisa, C., Saito-Kanatani, M., Maeda, K.I., Fujii, T., Osuga, Y., 2017. F4/80+ Macrophages Contribute to Clearance of Senescent Cells in the Mouse Postpartum Uterus. Endocrinology 158, 2344-2353.

Flowers, A., Bell-Temin, H., Jalloh, A., Stevens, S.M., Jr., Bickford, P.C., 2017. Proteomic anaysis of aged microglia: shifts in transcription, bioenergetics, and nutrient response. J. Neuroinflammation 14, 96.

Fong, C.C., Zhang, Q., Shi, Y.F., Wu, R.S., Fong, W.F., Yang, M., 2007. Effect of hypoxia on RAW264.7 macrophages apoptosis and signaling. Toxicology 235, 52-61.

Fuentes, L., Wouters, K., Hannou, S.A., Cudejko, C., Rigamonti, E., Mayi, T.H., Derudas, B., Pattou, F., Chinetti-Gbaguidi, G., Staels, B., Paumelle, R., 2011. Downregulation of the tumour suppressor $\mathrm{p} 16 \mathrm{INK} 4 \mathrm{~A}$ contributes to the polarisation of human macrophages toward an adipose tissue macrophage (ATM)-like phenotype. Diabetologia 54, 31503156.

Galván-Peña, S., O'Neill, L.A.J., 2014. Metabolic reprograming in macrophage polarization. Front. Immunol. 5, 420-420.

Gizard, F., Heywood, E.B., Findeisen, H.M., Zhao, Y., Jones, K.L., Cudejko, C., Post, G.R., Staels, B., Bruemmer, D., 2011. Telomerase Activation in Atherosclerosis and Induction of Telomerase Reverse Transcriptase Expression by Inflammatory Stimuli in Macrophages. Arteriosclerosis, Thrombosis, and Vascular Biology 31, 245-252.

Hall, B.M., Balan, V., Gleiberman, A.S., Strom, E., Krasnov, P., Virtuoso, L.P., Rydkina, E., Vujcic, S., Balan, K., Gitlin, I., Leonova, K., Polinsky, A., Chernova, O.B., Gudkov, A.V., 2016. Aging of mice is associated with p16(Ink4a)- and $\beta$-galactosidase-positive macrophage accumulation that can be induced in young mice by senescent cells. Aging (Albany N. Y.) 8, 1294-1315.

Hall, B.M., Balan, V., Gleiberman, A.S., Strom, E., Krasnov, P., Virtuoso, L.P., Rydkina, E., Vujcic, S., Balan, K., Gitlin, I.I., Leonova, K.I., Consiglio, C.R., Gollnick, S.O., Chernova, O.B., Gudkov, A.V., 2017. p16(Ink4a) and senescence-associated $\beta$ galactosidase can be induced in macrophages as part of a reversible response to physiological stimuli. Aging 9, 1867-1884.

Hayflick, L., 1998. How and why we age. Exp. Gerontol. 33, 639-653.

Hayflick, L., Moorhead, P.S., 1961. The serial cultivation of human diploid cell strains. Exp. Cell Res. 25, 585-621.

He, M., Chiang, H.-H., Luo, H., Zheng, Z., Qiao, Q., Wang, L., Tan, M., Ohkubo, R., Mu, W.C., Zhao, S., Wu, H., Chen, D., 2020. An Acetylation Switch of the NLRP3 Inflammasome Regulates Aging-Associated Chronic Inflammation and Insulin Resistance. Cell Metab. 31, 580-591.e585.

Hernandez-Segura, A., Nehme, J., Demaria, M., 2018. Hallmarks of Cellular Senescence. Trends Cell Biol. 28, 436-453. 
Herrero, C., Marqués, L., Lloberas, J., Celada, A., 2001. IFN-gamma-dependent transcription of MHC class II IA is impaired in macrophages from aged mice. The Journal of clinical investigation 107, 485-493.

Hickson, L.J., Langhi Prata, L.G.P., Bobart, S.A., Evans, T.K., Giorgadze, N., Hashmi, S.K., Herrmann, S.M., Jensen, M.D., Jia, Q., Jordan, K.L., Kellogg, T.A., Khosla, S., Koerber, D.M., Lagnado, A.B., Lawson, D.K., LeBrasseur, N.K., Lerman, L.O., McDonald, K.M., McKenzie, T.J., Passos, J.F., Pignolo, R.J., Pirtskhalava, T., Saadiq, I.M., Schaefer, K.K., Textor, S.C., Victorelli, S.G., Volkman, T.L., Xue, A., Wentworth, M.A., Wissler Gerdes, E.O., Zhu, Y., Tchkonia, T., Kirkland, J.L., 2019. Senolytics decrease senescent cells in humans: Preliminary report from a clinical trial of Dasatinib plus Quercetin in individuals with diabetic kidney disease. EBioMedicine 47, 446-456.

Holt, D.J., Grainger, D.W., 2012. Senescence and quiescence induced compromised function in cultured macrophages. Biomaterials 33, 7497-7507.

Huo, L., Magliano, D.J., Rancière, F., Harding, J.L., Nanayakkara, N., Shaw, J.E., Carstensen, B., 2018. Impact of age at diagnosis and duration of type 2 diabetes on mortality in Australia 1997-2011. Diabetologia 61, 1055-1063.

Imperatore, F., Maurizio, J., Vargas Aguilar, S., Busch, C.J., Favret, J., Kowenz-Leutz, E., Cathou, W., Gentek, R., Perrin, P., Leutz, A., Berruyer, C., Sieweke, M.H., 2017. SIRT1 regulates macrophage self-renewal. EMBO J. 36, 2353-2372.

Irvine, K.M., Skoien, R., Bokil, N.J., Melino, M., Thomas, G.P., Loo, D., Gabrielli, B., Hill, M.M., Sweet, M.J., Clouston, A.D., Powell, E.E., 2014. Senescent human hepatocytes express a unique secretory phenotype and promote macrophage migration. World J. Gastroenterol. 20, 17851-17862.

Kang, T.W., Yevsa, T., Woller, N., Hoenicke, L., Wuestefeld, T., Dauch, D., Hohmeyer, A., Gereke, M., Rudalska, R., Potapova, A., Iken, M., Vucur, M., Weiss, S., Heikenwalder, M., Khan, S., Gil, J., Bruder, D., Manns, M., Schirmacher, P., Tacke, F., Ott, M., Luedde, T., Longerich, T., Kubicka, S., Zender, L., 2011. Senescence surveillance of premalignant hepatocytes limits liver cancer development. Nature 479, 547-551.

Kang, Y., Zhang, H., Zhao, Y., Wang, Y., Wang, W., He, Y., Zhang, W., Zhang, W., Zhu, X., Zhou, Y., Zhang, L., Ju, Z., Shi, L., 2018. Telomere Dysfunction Disturbs Macrophage Mitochondrial Metabolism and the NLRP3 Inflammasome through the PGC1 $\alpha /$ TNFAIP3 Axis. Cell Rep. 22, 3493-3506.

Karin, O., Agrawal, A., Porat, Z., Krizhanovsky, V., Alon, U., 2019. Senescent cell turnover slows with age providing an explanation for the Gompertz law. Nature Communications 10, 5495.

Katzir, I., Adler, M., Karin, O., Mendelsohn-Cohen, N., Mayo, A., Alon, U., 2021. Senescent cells and the incidence of age-related diseases. Aging Cell 20, e13314.

Kay, M.M., 1975. Mechanism of removal of senescent cells by human macrophages in situ. Proc. Natl. Acad. Sci. U. S. A. 72, 3521-3525.

Kim, K.-H., Park, B., Rhee, D.-K., Pyo, S., 2015. Acrylamide Induces Senescence in Macrophages through a Process Involving ATF3, ROS, p38/JNK, and a TelomeraseIndependent Pathway. Chem. Res. Toxicol. 28, 71-86.

Krieger, M., Herz, J., 1994. Structures and functions of multiligand lipoprotein receptors: macrophage scavenger receptors and LDL receptor-related protein (LRP). Annu. Rev. Biochem. 63, 601-637. 
Krishnamurthy, J., Ramsey, M.R., Ligon, K.L., Torrice, C., Koh, A., Bonner-Weir, S., Sharpless, N.E., 2006. p16INK4a induces an age-dependent decline in islet regenerative potential. Nature 443, 453-457.

Krizhanovsky, V., Yon, M., Dickins, R.A., Hearn, S., Simon, J., Miething, C., Yee, H., Zender, L., Lowe, S.W., 2008. Senescence of activated stellate cells limits liver fibrosis. Cell 134, 657-667.

Kumar, R., Sharma, A., Padwad, Y., Sharma, R., 2020. Preadipocyte secretory factors differentially modulate murine macrophage functions during aging which are reversed by the application of phytochemical EGCG. Biogerontology 21, 325-343.

Kumari, R., Jat, P., 2021. Mechanisms of Cellular Senescence: Cell Cycle Arrest and Senescence Associated Secretory Phenotype. Frontiers in Cell and Developmental Biology 9.

Lacerda Mariano, L., Rousseau, M., Varet, H., Legendre, R., Gentek, R., Saenz Coronilla, J., Bajenoff, M., Gomez Perdiguero, E., Ingersoll, M.A., 2020. Functionally distinct resident macrophage subsets differentially shape responses to infection in the bladder. Sci Adv 6 .

Lee, K.-a., Robbins, P.D., Camell, C.D., 2021. Intersection of immunometabolism and immunosenescence during aging. Current Opinion in Pharmacology 57, 107-116.

Lewis-McDougall, F.C., Ruchaya, P.J., Domenjo-Vila, E., Shin Teoh, T., Prata, L., Cottle, B.J., Clark, J.E., Punjabi, P.P., Awad, W., Torella, D., Tchkonia, T., Kirkland, J.L., EllisonHughes, G.M., 2019. Aged-senescent cells contribute to impaired heart regeneration. Aging Cell 18, e12931.

Linehan, E., Dombrowski, Y., Snoddy, R., Fallon, P.G., Kissenpfennig, A., Fitzgerald, D.C., 2014. Aging impairs peritoneal but not bone marrow-derived macrophage phagocytosis. Aging Cell 13, 699-708.

López-Otín, C., Blasco, M.A., Partridge, L., Serrano, M., Kroemer, G., 2013. The hallmarks of aging. Cell 153, 1194-1217.

Ludlow, A.T., Spangenburg, E.E., Chin, E.R., Cheng, W.-H., Roth, S.M., 2014. Telomeres shorten in response to oxidative stress in mouse skeletal muscle fibers. The journals of gerontology. Series A, Biological sciences and medical sciences 69, 821-830.

Lujambio, A., Akkari, L., Simon, J., Grace, D., Tschaharganeh, D.F., Bolden, J.E., Zhao, Z., Thapar, V., Joyce, J.A., Krizhanovsky, V., Lowe, S.W., 2013. Non-cell-autonomous tumor suppression by p53. Cell 153, 449-460.

Maduro, A.T., Luís, C., Soares, R., 2021. Ageing, cellular senescence and the impact of diet: an overview. Porto Biomedical Journal 6, e120.

Mahbub, S., Deburghgraeve, C.R., Kovacs, E.J., 2012. Advanced age impairs macrophage polarization. J Interferon Cytokine Res 32, 18-26.

Mazucanti, C.H., Cabral-Costa, J.V., Vasconcelos, A.R., Andreotti, D.Z., Scavone, C., Kawamoto, E.M., 2015. Longevity Pathways (mTOR, SIRT, Insulin/IGF-1) as Key Modulatory Targets on Aging and Neurodegeneration. Curr. Top. Med. Chem. 15, 21162138.

Mazzoni, M., Mauro, G., Erreni, M., Romeo, P., Minna, E., Vizioli, M.G., Belgiovine, C., Rizzetti, M.G., Pagliardini, S., Avigni, R., Anania, M.C., Allavena, P., Borrello, M.G., Greco, A., 2019. Senescent thyrocytes and thyroid tumor cells induce M2-like macrophage polarization of human monocytes via a PGE2-dependent mechanism. J. Exp. Clin. Cancer Res. 38, 208. 
Mevorach, D., Trahtemberg, U., Krispin, A., Attalah, M., Zazoun, J., Tabib, A., Grau, A., Verbovetski-Reiner, I., 2010. What do we mean when we write "senescence," "apoptosis," "necrosis," or "clearance of dying cells"? Ann. N. Y. Acad. Sci. 1209, 1-9.

Minamino, T., Orimo, M., Shimizu, I., Kunieda, T., Yokoyama, M., Ito, T., Nojima, A., Nabetani, A., Oike, Y., Matsubara, H., Ishikawa, F., Komuro, I., 2009. A crucial role for adipose tissue p53 in the regulation of insulin resistance. Nat. Med. 15, 1082-1087.

Minhas, P.S., Liu, L., Moon, P.K., Joshi, A.U., Dove, C., Mhatre, S., Contrepois, K., Wang, Q., Lee, B.A., Coronado, M., Bernstein, D., Snyder, M.P., Migaud, M., Majeti, R., MochlyRosen, D., Rabinowitz, J.D., Andreasson, K.I., 2019. Macrophage de novo NAD(+) synthesis specifies immune function in aging and inflammation. Nat. Immunol. 20, 5063.

Minutti, C.M., Knipper, J.A., Allen, J.E., Zaiss, D.M., 2017. Tissue-specific contribution of macrophages to wound healing. Semin Cell Dev Biol 61, 3-11.

Mogensen, T.H., 2009. Pathogen recognition and inflammatory signaling in innate immune defenses. Clin. Microbiol. Rev. 22, 240-273.

Narzt, M.S., Pils, V., Kremslehner, C., Nagelreiter, I.M., Schosserer, M., Bessonova, E., Bayer, A., Reifschneider, R., Terlecki-Zaniewicz, L., Waidhofer-Söllner, P., Mildner, M., Tschachler, E., Cavinato, M., Wedel, S., Jansen-Dürr, P., Nanic, L., Rubelj, I., ElGhalbzouri, A., Zoratto, S., Marchetti-Deschmann, M., Grillari, J., Gruber, F., Lämmermann, I., 2021. Epilipidomics of Senescent Dermal Fibroblasts Identify Lysophosphatidylcholines as Pleiotropic Senescence-Associated Secretory Phenotype (SASP) Factors. J. Invest. Dermatol. 141, 993-1006.e1015.

Nyunoya, T., Powers, L.S., Yarovinsky, T.O., Butler, N.S., Monick, M.M., Hunninghake, G.W., 2003. Hyperoxia induces macrophage cell cycle arrest by adhesion-dependent induction of p21Cip1 and activation of the retinoblastoma protein. J. Biol. Chem. 278, 3609936106.

Ogata, Y., Yamada, T., Hasegawa, S., Sanada, A., Iwata, Y., Arima, M., Nakata, S., Sugiura, K., Akamatsu, H., 2021. SASP-induced macrophage dysfunction may contribute to accelerated senescent fibroblast accumulation in the dermis. Exp. Dermatol. 30, 84-91.

Ogrodnik, M., Evans, S.A., Fielder, E., Victorelli, S., Kruger, P., Salmonowicz, H., Weigand, B.M., Patel, A.D., Pirtskhalava, T., Inman, C.L., Johnson, K.O., Dickinson, S.L., Rocha, A., Schafer, M.J., Zhu, Y., Allison, D.B., von Zglinicki, T., LeBrasseur, N.K., Tchkonia, T., Neretti, N., Passos, J.F., Kirkland, J.L., Jurk, D., 2021. Whole-body senescent cell clearance alleviates age-related brain inflammation and cognitive impairment in mice. Aging Cell 20, e13296.

Orecchioni, M., Ghosheh, Y., Pramod, A.B., Ley, K., 2019. Macrophage Polarization: Different Gene Signatures in M1(LPS+) vs. Classically and M2(LPS-) vs. Alternatively Activated Macrophages. Front. Immunol. 10.

Ovadya, Y., Landsberger, T., Leins, H., Vadai, E., Gal, H., Biran, A., Yosef, R., Sagiv, A., Agrawal, A., Shapira, A., Windheim, J., Tsoory, M., Schirmbeck, R., Amit, I., Geiger, H., Krizhanovsky, V., 2018. Impaired immune surveillance accelerates accumulation of senescent cells and aging. Nature Communications 9, 5435.

Palmer, A.K., Xu, M., Zhu, Y., Pirtskhalava, T., Weivoda, M.M., Hachfeld, C.M., Prata, L.G., van Dijk, T.H., Verkade, E., Casaclang-Verzosa, G., Johnson, K.O., Cubro, H., Doornebal, E.J., Ogrodnik, M., Jurk, D., Jensen, M.D., Chini, E.N., Miller, J.D., Matveyenko, A., Stout, M.B., Schafer, M.J., White, T.A., Hickson, L.J., Demaria, M., 
Garovic, V., Grande, J., Arriaga, E.A., Kuipers, F., von Zglinicki, T., LeBrasseur, N.K., Campisi, J., Tchkonia, T., Kirkland, J.L., 2019. Targeting senescent cells alleviates obesity-induced metabolic dysfunction. Aging Cell 18, e12950.

Pereira, B.I., Devine, O.P., Vukmanovic-Stejic, M., Chambers, E.S., Subramanian, P., Patel, N., Virasami, A., Sebire, N.J., Kinsler, V., Valdovinos, A., LeSaux, C.J., Passos, J.F., Antoniou, A., Rustin, M.H.A., Campisi, J., Akbar, A.N., 2019. Senescent cells evade immune clearance via HLA-E-mediated NK and CD8+ T cell inhibition. Nature Communications 10, 2387.

Prata, L.G.P.L., Ovsyannikova, I.G., Tchkonia, T., Kirkland, J.L., 2018. Senescent cell clearance by the immune system: Emerging therapeutic opportunities. Semin. Immunol. 40, 101275.

Prattichizzo, F., De Nigris, V., Mancuso, E., Spiga, R., Giuliani, A., Matacchione, G., Lazzarini, R., Marcheselli, F., Recchioni, R., Testa, R., La Sala, L., Rippo, M.R., Procopio, A.D., Olivieri, F., Ceriello, A., 2018. Short-term sustained hyperglycaemia fosters an archetypal senescence-associated secretory phenotype in endothelial cells and macrophages. Redox biology 15, 170-181.

Renshaw, M., Rockwell, J., Engleman, C., Gewirtz, A., Katz, J., Sambhara, S., 2002. Cutting edge: impaired Toll-like receptor expression and function in aging. J. Immunol. 169, 4697-4701.

Report, H.o.L., 2021. Ageing: Science, Technology and Healthy Living, UK.

Robinson, A.R., Yousefzadeh, M.J., Rozgaja, T.A., Wang, J., Li, X., Tilstra, J.S., Feldman, C.H., Gregg, S.Q., Johnson, C.H., Skoda, E.M., Frantz, M.-C., Bell-Temin, H., PopeVarsalona, H., Gurkar, A.U., Nasto, L.A., Robinson, R.A.S., Fuhrmann-Stroissnigg, H., Czerwinska, J., McGowan, S.J., Cantu-Medellin, N., Harris, J.B., Maniar, S., Ross, M.A., Trussoni, C.E., LaRusso, N.F., Cifuentes-Pagano, E., Pagano, P.J., Tudek, B., Vo, N.V., Rigatti, L.H., Opresko, P.L., Stolz, D.B., Watkins, S.C., Burd, C.E., Croix, C.M.S., Siuzdak, G., Yates, N.A., Robbins, P.D., Wang, Y., Wipf, P., Kelley, E.E., Niedernhofer, L.J., 2018. Spontaneous DNA damage to the nuclear genome promotes senescence, redox imbalance and aging. Redox Biology 17, 259-273.

Rodriguez-Menocal, L., Faridi, M.H., Martinez, L., Shehadeh, L.A., Duque, J.C., Wei, Y., Mesa, A., Pena, A., Gupta, V., Pham, S.M., Vazquez-Padron, R.I., 2014. Macrophage-derived IL-18 and increased fibrinogen deposition are age-related inflammatory signatures of vascular remodeling. American Journal of Physiology-Heart and Circulatory Physiology 306, H641-H653.

Röszer, T., 2015. Understanding the Mysterious M2 Macrophage through Activation Markers and Effector Mechanisms. Mediators Inflamm. 2015, 816460.

Sagiv, A., Burton, D.G.A., Moshayev, Z., Vadai, E., Wensveen, F., Ben-Dor, S., Golani, O., Polic, B., Krizhanovsky, V., 2016. NKG2D ligands mediate immunosurveillance of senescent cells. Aging 8, 328-344.

Sampson, M.J., Winterbone, M.S., Hughes, J.C., Dozio, N., Hughes, D.A., 2006. Monocyte telomere shortening and oxidative DNA damage in type 2 diabetes. Diabetes Care 29, 283-289.

Schliehe, C., Redaelli, C., Engelhardt, S., Fehlings, M., Mueller, M., van Rooijen, N., Thiry, M., Hildner, K., Weller, H., Groettrup, M., 2011. CD8- dendritic cells and macrophages cross-present poly(D,L-lactate-co-glycolate) acid microsphere-encapsulated antigen in vivo. J. Immunol. 187, 2112-2121. 
Sebastián, C., Herrero, C., Serra, M., Lloberas, J., Blasco, M.A., Celada, A., 2009. Telomere Shortening and Oxidative Stress in Aged Macrophages Results in Impaired STAT5a Phosphorylation. The Journal of Immunology 183, 2356-2364.

Sedelnikova, O.A., Horikawa, I., Zimonjic, D.B., Popescu, N.C., Bonner, W.M., Barrett, J.C., 2004. Senescing human cells and ageing mice accumulate DNA lesions with unrepairable double-strand breaks. Nat. Cell Biol. 6, 168-170.

Sen, B., Aggarwal, S., Nath, R., Sehgal, R., Rastogi, A., Trehanpati, N., Ramakrishna, G., 2021. Secretome of senescent hepatoma cells modulate macrophage polarization and neutrophil extracellular traps formation. bioRxiv, 2020.2012.2030.423905.

Sharma, R., Kapila, R., Haq, M.R., Salingati, V., Kapasiya, M., Kapila, S., 2014. Age-associated aberrations in mouse cellular and humoral immune responses. Aging Clin. Exp. Res. 26, 353-362.

Sharma, R., Kapila, R., Kapila, S., 2013. Probiotics as Anti-immunosenescence Agents. Food Rev. Int. 29, 201-216.

Sharma, R., Kumar, R., Sharma, A., Goel, A., Padwad, Y., 2021. Long term consumption of green tea EGCG enhances healthspan and lifespan in mice by mitigating multiple aspects of cellular senescence in mitotic and post-mitotic tissues, gut dysbiosis and immunosenescence. bioRxiv, 2021.2001.2001.425058.

Sharma, R., Padwad, Y., 2020. Perspectives of the potential implications of polyphenols in influencing the interrelationship between oxi-inflammatory stress, cellular senescence and immunosenescence during aging. Trends Food Sci. Technol. 98, 41-52.

Singh, M.V., Kotla, S., Le, N.T., Ae Ko, K., Heo, K.S., Wang, Y., Fujii, Y., Thi Vu, H., McBeath, E., Thomas, T.N., Jin Gi, Y., Tao, Y., Medina, J.L., Taunton, J., Carson, N., Dogra, V., Doyley, M.M., Tyrell, A., Lu, W., Qiu, X., Stirpe, N.E., Gates, K.J., Hurley, C., Fujiwara, K., Maggirwar, S.B., Schifitto, G., Abe, J.I., 2019. Senescent Phenotype Induced by p90RSK-NRF2 Signaling Sensitizes Monocytes and Macrophages to Oxidative Stress in HIV-Positive Individuals. Circulation 139, 1199-1216.

Spadaro, O., Camell, C.D., Bosurgi, L., Nguyen, K.Y., Youm, Y.H., Rothlin, C.V., Dixit, V.D., 2017. IGF1 Shapes Macrophage Activation in Response to Immunometabolic Challenge. Cell Rep. 19, 225-234.

Swift, M.E., Burns, A.L., Gray, K.L., DiPietro, L.A., 2001. Age-related alterations in the inflammatory response to dermal injury. J. Invest. Dermatol. 117, 1027-1035.

Tuttle, C.S.L., Waaijer, M.E.C., Slee-Valentijn, M.S., Stijnen, T., Westendorp, R., Maier, A.B., 2020. Cellular senescence and chronological age in various human tissues: A systematic review and meta-analysis. Aging Cell 19, e13083.

Vallejo, A.N., Brandes, J.C., Weyand, C.M., Goronzy, J.J., 1999. Modulation of CD28 expression: distinct regulatory pathways during activation and replicative senescence. $\mathrm{J}$. Immunol. 162, 6572-6579.

van Beek, A.A., Van den Bossche, J., Mastroberardino, P.G., de Winther, M.P.J., Leenen, P.J.M., 2019. Metabolic Alterations in Aging Macrophages: Ingredients for Inflammaging? Trends Immunol. 40, 113-127.

Vicente, R., Mausset-Bonnefont, A.-L., Jorgensen, C., Louis-Plence, P., Brondello, J.-M., 2016. Cellular senescence impact on immune cell fate and function. Aging Cell 15, 400-406. von Zglinicki, T., 2002. Oxidative stress shortens telomeres. Trends Biochem. Sci. 27, 339-344. 
Wang, B., Zhong, Y., Li, Q., Cui, L., Huang, G., 2018. Autophagy of macrophages is regulated by PI3k/Akt/mTOR signalling in the development of diabetic encephalopathy. Aging (Albany N. Y.) 10, 2772-2782.

Wang, H., Fu, H., Zhu, R., Wu, X., Ji, X., Li, X., Jiang, H., Lin, Z., Tang, X., Sun, S., Chen, J., Wang, X., Li, Q., Ji, Y., Chen, H., 2020. BRD4 contributes to LPS-induced macrophage senescence and promotes progression of atherosclerosis-associated lipid uptake. Aging 12, 9240-9259.

Wissler Gerdes, E.O., Zhu, Y., Tchkonia, T., Kirkland, J.L., 2020. Discovery, development, and future application of senolytics: theories and predictions. The FEBS Journal 287, 24182427.

Wong, C.K., Smith, C.A., Sakamoto, K., Kaminski, N., Koff, J.L., Goldstein, D.R., 2017. Aging Impairs Alveolar Macrophage Phagocytosis and Increases Influenza-Induced Mortality in Mice. The Journal of Immunology 199, 1060-1068.

Wu, D., Lewis, E.D., Pae, M., Meydani, S.N., 2019. Nutritional Modulation of Immune Function: Analysis of Evidence, Mechanisms, and Clinical Relevance. Front. Immunol. 9.

Wynn, T.A., Chawla, A., Pollard, J.W., 2013. Macrophage biology in development, homeostasis and disease. Nature 496, 445-455.

Xu, M., Pirtskhalava, T., Farr, J.N., Weigand, B.M., Palmer, A.K., Weivoda, M.M., Inman, C.L., Ogrodnik, M.B., Hachfeld, C.M., Fraser, D.G., Onken, J.L., Johnson, K.O., Verzosa, G.C., Langhi, L.G.P., Weigl, M., Giorgadze, N., LeBrasseur, N.K., Miller, J.D., Jurk, D., Singh, R.J., Allison, D.B., Ejima, K., Hubbard, G.B., Ikeno, Y., Cubro, H., Garovic, V.D., Hou, X., Weroha, S.J., Robbins, P.D., Niedernhofer, L.J., Khosla, S., Tchkonia, T., Kirkland, J.L., 2018. Senolytics improve physical function and increase lifespan in old age. Nat. Med. 24, 1246-1256.

Yoshizaki, T., Schenk, S., Imamura, T., Babendure, J.L., Sonoda, N., Bae, E.J., Oh, D.Y., Lu, M., Milne, J.C., Westphal, C., Bandyopadhyay, G., Olefsky, J.M., 2010. SIRT1 inhibits inflammatory pathways in macrophages and modulates insulin sensitivity. American journal of physiology. Endocrinology and metabolism 298, E419-E428.

Zandi, S., Nakao, S., Chun, K.-H., Fiorina, P., Sun, D., Arita, R., Zhao, M., Kim, E., Schueller, O., Campbell, S., Taher, M., Melhorn, M.I., Schering, A., Gatti, F., Tezza, S., Xie, F., Vergani, A., Yoshida, S., Ishikawa, K., Yamaguchi, M., Sasaki, F., Schmidt-Ullrich, R., Hata, Y., Enaida, H., Yuzawa, M., Yokomizo, T., Kim, Y.-B., Sweetnam, P., Ishibashi, T., Hafezi-Moghadam, A., 2015. ROCK-isoform-specific polarization of macrophages associated with age-related macular degeneration. Cell Rep. 10, 1173-1186.

Zhou, D., Borsa, M., Simon, A.K., 2021. Hallmarks and detection techniques of cellular senescence and cellular ageing in immune cells. Aging Cell 20, e13316. 